\title{
Checklist of rotifer species from Albania (phylum Rotifera)
}

\author{
S. SHUMKA \\ Spase Shumka, Faculty of Biotechnology and Food, Agricultural University of Tirana, Tirana, Albania \\ ORCID: 0000-0002-5930-6034,Email: sprespa@gmail.com
}

\begin{abstract}
The checklist of Rotifera species recorded for Albanian inland waters and its neighboring regions is provided. A total of 140 species of bdelloids and monogononts, with representatives of 38 genera are listed. The history of rotifer surveys as a component of zooplankton in Albania started at the end of $19^{\text {th }}$ century. Mostly they were taxonomic and descriptive ones, while later on after the mid $20^{\text {th }}$ century appeared the systematic approaches emphasizing the particularities and richness of zooplankton in specific karstic Mediterranean ecosystems. In this article 140 taxa of bdelloids and monogononts representing 39 genera are reported.
\end{abstract}

Keywords. Rotifera, Albania, checklist, inland water, distribution records

\section{INTRODUCTION}

A lbania is a small country (Fig. 1) however, it is rich in water resources including lakes, rivers and springs. There are more than 152 streams and small rivers flow into the seven large rivers, Buna, Drini, Mati, Erzeni, Shkumbini, Semani, and Vjosa, which run southeast to north west towards the Adriatic coast (Cullaj et al. 2005). About 247 natural lakes are dispersed throughout the country, most of karstic or glacial origin and they are often very small (less than 1 ha). Albania shares with neighboring countries three of the largest Balkanic Lakes (Ohrid, Prespa and Shkodra). They represent distinct environments among all aquatic habitats of the Balkan in the view of origin, hydrology, and biodiversity (Shumka et al. 2018). Around 134 glacial lakes are situated mainly in the northeastern part of the country at altitudes between $1500-1800 \mathrm{~m}$ a.s.l. Generally, they are small, formed mainly over magmatic (mainly of ultrabasics) and terrene formations (Cullaj et al. 2005).

Following Segers (2007) the Phylum Rotifera comprises about 2030 known species worldwide and classified in three main groups, the marine Seisonida (4 species), the Monogononta (1570 species) and the unique, exclusively parthenogenetic Bdelloidea with 461 clonal species. Although the morphology of different species varies widely, all of them possess a specialized masticatory organ containing a set of densely sclerotized trophi (Fontaneto et al. 2008). Rotifers, as a component of zooplankton organisms, comprise crucial elements of the structure and function of freshwater ecosystems, not only as consumers of algae, bacteria, protozoans and other invertebrates (Russell et al. 2010), but also as food items for juvenile stages of several fish species (Shumka et al. 2018). Moreover, their pivotal role in freshwater ecosystems food web, as well as its sensitivity to both man-made and natural changes, makes zooplankton quite suitable for assessing alterations in the trophic dynamics and the ecological state of aquatic ecosystems related to changes in nutrient loading and climate (Hoffmann 1977, Ferrara et al. 2002, Preston \& Rusak 2010).

Similarly to other large Balkanic lakes like Ohrid and Prespa, the history of zooplankton surveys in Albania starts from the end of $19^{\text {th }}$ century. Basically they were taxonomic and descriptive ones, while later, after the mid $20^{\text {th }}$ century appeared the systematic approach emphasizing 


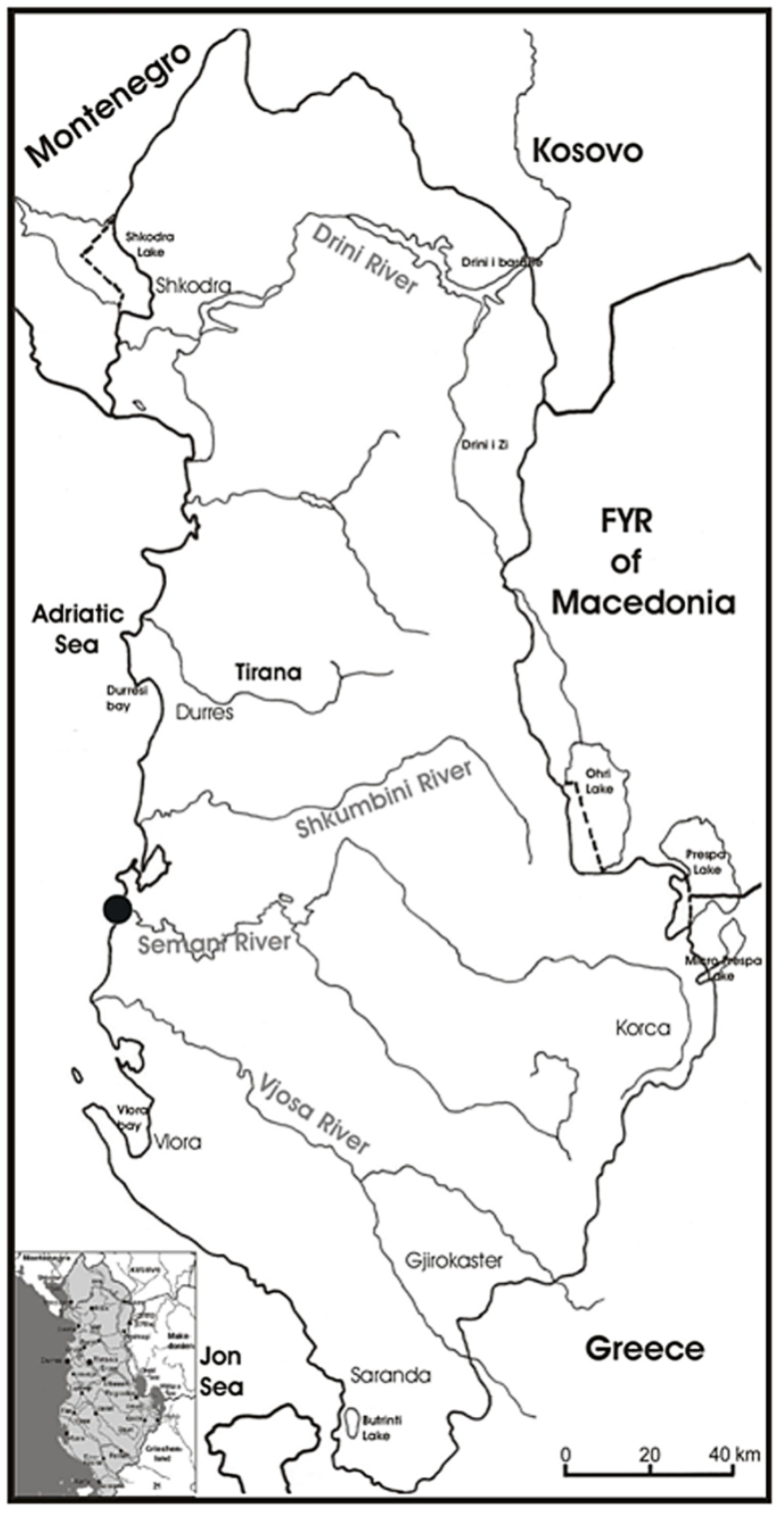

Figure 1. Map of Albania

the particularities and richness of zooplankton in specific karstic Mediterranean ecosystem. Amongst them can be mention Richard (1897) with his work dedicated to Cladocera, Steur (1900) with paper published on Copepoda, Vereščagin (1912) and Parenzan (193) on Cladocera, Nedeljković (1959) on Rotifera, Petkovski (1961) with fundamental work on Cladocera and Copeoda, separately Harpacticoida and Ostracoda, Živković (1965) and Milovanović \& Živković (1965) on Rotifera, Protozoa and Cladocera.

\section{MATERIALS AND METHODS}

There are a large number of references, including identification keys with abundant information on ecological and systematical aspects of rotifers from different regions of the world. From this, the following papers were consulted: Segers (2002) and Wallace et al. (2006); higher-level classification. For taxonomy and distribution of the different families the following sources were used: De Ridder (1986, 1991, 1993), De Ridder \& Segers (1997), Donner (1965), De Smet (2006), De Smet \& Pourriot (1997), Jersabek (2003), Koste (1978), Koste \& Shiel (1987, 1989a, b), Nogrady et al., (1995), Nogrady \& Segers (2002), Segers (1995a, b, 2003), Segers \& Wallace (2001), Sørensen et al. (2005).

The following articles were used to compile the list of rotifers of Albanian inland waters: Brusina (1898), Byron (1981), Gannon \& Stemberger (1981), Guseska (2012), Guseska et al. (2008, 2012, 2014), Gushevska et al. (1996), Kiefer (1937), Kostoski (1998), Kostoski et al. (2004, 2005), Michaloudi (2005), Michaloudi et al. (1997), Milovanovic \& Živkovic (1965), Nedeljkovic (1959), Parenzan (1931), Petković (1973, 1975, 1978, 1981), Popovska-Stankovič et al. (1988, 2003), Richard (1897), Serafimova-Hadzisce $(1954,1958,1975)$, Shumka $(1994,1997 a, b$, 2000, 2001, 2014), Shumka \& Miho (2006), Shumka \& Nikleka (2018), Shumka \& Špoljar (2018), Shumka et al. (1998, 2018), Steuer (1900), Tasevska (2002), Tasevska et al. (2006, 2008, 2012a, b, 2017, 2018), Veršcagin (1912), Živkovic (1974, 1975),

\section{RESULTS AND DISCUSSION}

All species names appeared in the literature listed above were checked and corrected based on Segers et al. 2007 and Jersabek \& Leitner 2013, and the following changes were made: Brachionus calyciflorus $f$. amphiceros (Ehrenberg, 1838) (synonym, considered an infrasubspecific variant of B. calyiflorus); Brachionus q. f. brevispinus Ehrenberg, 1832 (synonym, considered an infra 
subspecific variant of $B$. quadridentatus); Brachinus q. f. cluniorbicularis Skorikov, 1894 (synonym considered an infrasubspecific variant of B. quadridentatus); Brachionus quadridentatus $\mathrm{f}$. rhenanus Lauterborn, 1893, (synonym considered an infrasubspecific variant of B. quadridentatus); Lophocharis salpina (Ehrenberg, 1834) synonym of Lepadella salpina Ehrenberg, 1834; Testudinella patina trilobata (Anderson et Shepard, 1892), synonym of T. patina (Hermann 1783); Testudinella pseudoelliptica Bartoš, 1951 synonym of T. patina (Hermann 1783); Trichocerca similis similis (Wierzejski, 1893) synonymous with Rattulus bicornis (Western, 1893).

Reviewing the studies dedicated to the Albanian inland water Rotifera fauna is resulted in a list of 140 taxa of bdelloids and monogononts (Table 1). Among the rotifers, the most frequently reported genera are Lecane with 16 species, Trichocerca with 15 species, Brachionus (15 species), Keratella (7 species), Polyarthra (7 species) and Lepadella (6 species). At regional scale it is worth mentioning that from the neighboring Montenegro Petković $(1973,1978)$ recorded 205 rotifer taxa just for the Lake Scadar/Shkodra, while from North Macedonia for the lakes Ohrid,
Prespa and Dojran altogether 60 rotifer taxa were reported (Tasevska et al. 2006). However, the high number of species reported for the Lake Scadar/Shkodra by Petković $(1973,1978)$ does not reflect the latest taxonomic results of Segers et al., (2007) and Jersabek \& Leitner (2013) listing numerous synonym names and corrections.

The biogeography of Rotifera is highly controversial and prior to the work of Dumont (1983) it was generally accepted that all taxa were cosmopolitan. In the present list of the Albanian rotifers most of the species show a typical Palearctic distribution (Segers 2007) however, to clear the biogeography of several widely distributed species further studies with molecular approaches are needed.

Based on the data presented in the Table 1 almost $90 \%$ of the species are found in the Drini Basin (including Lakes of Lesser and Macro Prespa, Ohrid and Scadar/Shkodra), while the transitional water bodies belonging to Ionian and Adriatic Coastal areas are populated only by 9 different species. This disproportional distribution of species calls for further studies.

Table 1. List of Bdelloidea and Monogononta rotifers recorded from continental fresh- and transitional water habitats in Albania (genus and species)

\begin{tabular}{|c|c|c|}
\hline Taxon & Locality (water basins) & References \\
\hline \multicolumn{3}{|l|}{ Adineta Hudson \& Gosse, 1886} \\
\hline A. steineri Bartos, 1951 & A5 (A) & $14,15,30,31,32,38,40,41,42$ \\
\hline \multicolumn{3}{|l|}{ Anuraeopsis Lauterborn, 1900} \\
\hline A. fissa Gosse, 1851 & $\mathbf{A 1}, \mathbf{A 2}, \mathbf{A 5}(\mathrm{A})$ & $12,13,15,17$ \\
\hline A. coelata de Beauchamp, 1932 & A5 (A) & $12,13,15,17,30,31,32$ \\
\hline \multicolumn{3}{|l|}{ Ascomorpha Perty, 1850} \\
\hline A. ecaudis Perty, 1850 & A5, B1, B2 (A, B) & $12,13,15,17,30,31,32$ \\
\hline A. ovalis Carlin, 1943 & A2, A5, B1 (A, B) & \\
\hline A. saltans Bartsch, 1870 & $\mathbf{A 5}(\mathrm{A})$ & $12,13,14,15,30,31,32,38,40,41,42$ \\
\hline \multicolumn{3}{|l|}{ Asplanchna Gosse, 1850} \\
\hline A. girodide Guerne, 1888 & A5 (A) & $14,15,30,31,32,38,40,41,42$ \\
\hline A. priodonta Gosse, 1850 & $\mathrm{~A} 1, \mathrm{~A} 2, \mathrm{~A} 3, \mathrm{~A} 4, \mathrm{A5}, \mathrm{A6}, \mathrm{B} 1, \mathrm{~B} 2, \mathrm{C} 1$ & \\
\hline A. sieboldii Leydig, 1854 & $\operatorname{A5}(\mathrm{A})$ & $12,13,14,15,30,31,32,38,40,41,42$ \\
\hline \multicolumn{3}{|l|}{ Brachionus Pallas, 1766} \\
\hline B. angularis Gosse, 1851 & $\begin{array}{l}\text { A1, A2, A3, A5, B1, B2, C1, C2, C2, } \\
\text { C4, C5, D1 (A, B, C, D) }\end{array}$ & $\begin{array}{l}3,4,5,6,7,8,9,10,11,12,13,14,15 \\
30,31,32,38,40,41,42\end{array}$ \\
\hline B. bidentatus Anderson, 1889 & A5 (A) & $13,14,15,30,31,32,38,40,41,42$ \\
\hline B. calyciflorus Pallas, 1776 & A5, B2 (A, B) & $12,13,14,15,28,29,31,32,38,40$ \\
\hline B. dimidiatus Bryce, 1931 & $\mathbf{A 5}(\mathrm{A})$ & $\begin{array}{l}12,13,14,15,17,30,31,32,38,40,41 \text {, } \\
42\end{array}$ \\
\hline
\end{tabular}




\begin{tabular}{|c|c|c|}
\hline B. diversicornis Daday, 1883 & $\mathrm{A1}, \mathbf{A 2}, \mathbf{A 5}(\mathrm{A})$ & $2,3,10,11,18,19,20,21,22,23,24,25$ \\
\hline B. falcatus Zacharias, 1898 & A5 (A) & $12,13,31,32,42$ \\
\hline B. forficula Wierzejski, 1891 & A1, A2, A5 (A) & $13,14,15,31,32,38,42$ \\
\hline B. havanaensis Rousselet, 1911 & A5 (A) & $13,14,15,31,32,38,42$ \\
\hline B. leydigii Cohn, 1862 & A5 (A) & $13,14,15,31,32,38,42$ \\
\hline B. plicatilis O.F. Müller 1786 & A5, B1 (A, B) & $12,13,14,15,28,29,31,32,38,40$ \\
\hline B. rhenanus Lauterborn, 1893 . & $\mathrm{A5}, \mathrm{B1}(\mathrm{A}, \mathrm{B})$ & $12,13,14,15,28,29,31$ \\
\hline B. quadridentatus Hermann, 1783 & $\mathrm{A1}, \mathrm{A3}, \mathrm{A} 4, \mathrm{A5}, \mathrm{B2} .(\mathrm{A}, \mathrm{B})$ & $12,13,14,15,28,29,31,32,38,40$ \\
\hline B. brevispinus Ehrenberg, 1832 & A5 (A) & $13,14,15,31,32,38,42$ \\
\hline $\begin{array}{l}\text { B. quadridentatus melhemi Barrios } \\
\text { et Daday, } 1894\end{array}$ & A5 (A) & $13,14,15,31,32,38,42$ \\
\hline B. urceolaris O.F. Müller, 1773 & A2, A5 (A) & $13,14,15,31,32,38,42$ \\
\hline \multicolumn{3}{|l|}{$\begin{array}{l}\text { Cephalodella Bory de St. Vincent, } \\
1826\end{array}$} \\
\hline C. catellina O.F.Müller, 1786 & A2, A5 (A) & $13,14,15,31,32,38,39,40,41,42$ \\
\hline C. forficula Ehrenberg, 1831 & $\mathbf{A 2}, \mathbf{A 5}(\mathrm{A})$ & $13,14,15,31,32,38,39,40,41,42$ \\
\hline C. gibba Ehrenberg, 1832 & $\mathbf{A 2}, \mathbf{A 3}, \mathbf{A 5}(\mathrm{A})$ & $10,11,18,19,20,21,22$ \\
\hline C. misgurnus Wulfert, 1937 & A5. (A) & $13,14,15,31,32,38,42$ \\
\hline C. ventripes Dixon-Nuttall, 1901 & A5, C1 (A, C) & $13,14,15,29,31,32,38,42$ \\
\hline \multicolumn{3}{|l|}{ Collotheca Harring, 1913} \\
\hline C. mutabilis Hudson, 1885 & A5 (A) & $13,14,15,31,32,38,42$ \\
\hline C. pelagic Rousselet, 1893 & A5 (A) & $13,14,15,31,32,38,42$ \\
\hline \multicolumn{3}{|l|}{ Colurella Bory de St. Vincent, 1824} \\
\hline C. adriatica Ehrenberg, 1831 & $\begin{array}{l}\text { A3, A5, A6, D1, E1, E2, E3, E4 (A, } \\
D, E)\end{array}$ & $\begin{array}{l}3,4,5,6,7,8,9,10,11,12,13,14,15 \\
30,31,32,38,40,41,42\end{array}$ \\
\hline C. colurus Ehrenberg, 1830 & $\mathbf{A 2}, \mathbf{A 5}(\mathrm{A})$ & $13,14,15,31,32,38,39,40,41,42$ \\
\hline C. obtuse Gosse, 1886 & $\mathrm{A2}, \mathrm{A5}, \mathrm{A6}(\mathrm{A})$ & $13,14,15,31,32,38,39,40,41,42$ \\
\hline $\begin{array}{l}\text { C. uncinato bicuspidato Ehrenberg, } \\
1830\end{array}$ & A2, A3 (A) & $10,11,18,19,20,21,22,23,33$ \\
\hline C. uncinata O. F. Müller, 1773 & A5 (A) & $13,14,15,31,32,38,42$ \\
\hline \multicolumn{3}{|l|}{ Conochilus Ehrenberg, 1834} \\
\hline C. exiguous Ahlstrom, 1938 & A5 (A) & $13,14,15,31,32,38,42$ \\
\hline C. hippocrepis Schrank, 1830 & $\mathrm{A1}, \mathbf{A 2}, \mathbf{A 5}(\mathrm{A})$ & $13,14,15,31,32,38,39,40,41,42$ \\
\hline C. unicornis Rousselet, 1892 & A5 (A) & $13,14,15,31,32,42$ \\
\hline \multicolumn{3}{|l|}{ Dicranophorus Nitsch, 1827} \\
\hline D. forcipatus O.F. Müller, 1786 & A2, E1 (A, E) & $10,11,31$ \\
\hline D. grandis Ehrenberg, 1832 & $\mathbf{A 5}(\mathrm{A})$ & $14,15,30,31,32,38,40,41,42$ \\
\hline $\begin{array}{l}\text { D. rostratus Dixon Nuttal et } \\
\text { Freeman, } 1902\end{array}$ & A2, A5 (A) & $13,14,15,31,32,38,39,40,41,42$ \\
\hline \multicolumn{3}{|l|}{ Dipleuchlanis de Beauchamp, 1910} \\
\hline D. propatula Gosse, 1886 & A5 (A) & $13,14,15,31,32,38,39,40,41,42$ \\
\hline \multicolumn{3}{|l|}{ Dissotrocha Bryce, 1910} \\
\hline D. aculeata Ehrenberg, 1832 & $\mathrm{A3}, \mathrm{A5}, \mathrm{A6}(\mathrm{A})$ & $13,14,15,31,32,38,39,40,41,42$ \\
\hline \multicolumn{3}{|l|}{ Epiphanes Ehrenberg, 1832} \\
\hline $\begin{array}{l}\text { E. macrourus Barrois \&Daday, } \\
1894\end{array}$ & A5, B1 (A, B) & $13,14,15,31,32,38,39,40,41,42$ \\
\hline E. senta O.F. Müller, 1773 & A5, B1 (A, B) & $13,14,15,31,32,38,39,40,41,42$ \\
\hline \multicolumn{3}{|l|}{ Euchlanis Ehrenberg, 1832} \\
\hline E. dilatata Ehrenberg, 1832 & A2,A3, A5 (A) & $13,14,15,31,32,38,39,40,41,42$ \\
\hline E. incise Carlin, 1939 & A3, A5 (A) & $13,14,15,31,32,38,39,41,42$ \\
\hline E. meneta Myers, 1930 & A5 (A) & 39,41 \\
\hline \multicolumn{3}{|l|}{ Filinia Bory de St. Vincent, 1824} \\
\hline$F$. longiseta Ehrenberg, 1834 & $\begin{array}{l}\text { A1, A2, A3, A4, A5, B1, B2, C4, C5, } \\
\text { D1 (A, B, C, D) }\end{array}$ & $\begin{array}{l}2,3,4,6,7,8,9,10,12,13,14,15,19, \\
20,31,32,38,39,40,41,42\end{array}$ \\
\hline F.opoliensis Zacharias, 1898 & A5 (A) & $13,14,15,31,32,38,39,40,41,42$ \\
\hline F.terminalis Plate, 1886 & A5 (A) & $13,14,15,31,32,38,39,40,41,42$ \\
\hline
\end{tabular}




\begin{tabular}{|c|c|c|}
\hline Gastropus Imhof, 1898 & & \\
\hline G. hyptopus Ehrenberg, 1838 & A5 (A) & $13,14,15,31,32,38,39,40,41,42$ \\
\hline G.stylifer Imhof, 1891 & $\begin{array}{l}\text { A1, A2, A4, A5, B1, D1, E1 (A, B, } \\
\text { D, E) }\end{array}$ & $\begin{array}{l}3,4,5,6,7,8,9,10,11,12,13,14,15 \\
30,31,32,38,40,41,42\end{array}$ \\
\hline \multicolumn{3}{|l|}{ Hexarthra Schmarda, 1854} \\
\hline H. mira Hudson, 1871 & A5 (A) & $13,14,15,31,32,38,39,40,41,42$ \\
\hline \multicolumn{3}{|l|}{ Kellicottia Ahlstrom, 1938} \\
\hline K. longispina Kellicott, 1879 & $\begin{array}{l}\mathrm{A1}, \mathrm{A2}, \mathrm{A3}, \mathrm{A4}, \mathrm{A5}, \mathrm{B} 1, \mathrm{~B} 2, \mathrm{C} 1, \\
\mathrm{C} 2, \mathrm{C} 3, \mathrm{C} 4, \mathrm{D} 1, \mathrm{E} 1 \text { (A, B, C, D, E) }\end{array}$ & $\begin{array}{l}2,3,4,6,7,8,9,10,12,13,14,15,28, \\
19,20,21,22,23,24,25,27,28,29,30, \\
31,32,38,39,40,41,42\end{array}$ \\
\hline \multicolumn{3}{|l|}{ Keratella Bory de St. Vincent, 1822} \\
\hline K. cochlearis Gosse, 1851 & $\begin{array}{l}\text { A1, A2, A3, A4, A5, B1, B2, C1, C2, } \\
\text { C3, C4, C5, D1, E2 (A, B, C, D, E) }\end{array}$ & $\begin{array}{l}2,3,4,6,7,8,9,10,12,13,14,15,19, \\
20,21,22,23,24,25,27,28,29,30,31, \\
32,38,39,40,41,42\end{array}$ \\
\hline $\begin{array}{l}\text { K. cochlearis } v \text {. hispida Lauterborn, } \\
1898\end{array}$ & A2 (A) & $2,25,26,36$ \\
\hline K. hiemalis Carlin, 1943 & A5 (A) & $13,14,15,31,32,38,39,40,41,42$ \\
\hline K. quadrata O.F. Müller, 1786 , & $\mathbf{A 1}, \mathbf{A 2}, \mathbf{A 5}, \mathrm{D} 1, \mathbf{E 2}(\mathrm{A}, \mathrm{D}, \mathrm{E})$ & $2,3,4,6,7,8,9,10,12,13,14,15$ \\
\hline K. tecta Gosse, 1851 & $\mathbf{A 2}, \mathbf{A 5}(\mathrm{A})$ & $13,14,15,31,32,38,39,40,41,42$ \\
\hline K. ticinensis Callerio, 1921 & A5 (A) & $13,14,15,31,32,38,39,40,41,42$ \\
\hline K. valga Ehrenberg, 1832 & A5 (A) & $13,14,15,31,32,38,39,40,41,42$ \\
\hline \multicolumn{3}{|l|}{ Lecane Nitzsch, 1827} \\
\hline L. bulla Gosse, 1851 & $\mathrm{A2}, \mathbf{A 3}, \mathbf{A 5}(\mathrm{A})$ & $13,14,15,31,32,38,39,40,41,42$ \\
\hline L. closterocerca Schmarda, 1859 & A2, A3, A5, B1 (A, B) & $2,13,14,15,32,38,39,41,42$ \\
\hline L. copeis Harring et Myers, 1926 & $\mathbf{A 2}, \mathbf{A 5}(\mathrm{A})$ & $13,14,15,31,32,38,39,40,41,42$ \\
\hline L.curvirostris Yamamoto, 1941 & A5 (A) & $13,14,15,31,32,38,39,40,41,42$ \\
\hline L.elasma Harring\& Myers, 1926 & A5 (A) & $13,14,15,31,32,38,39,40,41,42$ \\
\hline L.elsa Nitzsch, 1827 & $\mathrm{A5}, \mathrm{A6}(\mathrm{A})$ & $13,14,15,31,32,38,39,40,41,42$ \\
\hline L.flexilis Gosse, 1886 & A5 (A) & $13,14,15,31,32,38,39,40,41,42$ \\
\hline L. hamata Stokes, 1896 & $\mathbf{A 5}(\mathrm{A})$ & $13,14,15,31,32,38,39,40,41,42$ \\
\hline L. ivli Wiszniewski, 1935 & A2, A5 (A) & $2,13,14,15,31,32,38,39,40,41,42$ \\
\hline L. lamellate Daday, 1893 & $\mathrm{A5}(\mathrm{A})$ & $13,14,15,31,32,38,39,40,41,42$ \\
\hline L.luna O.F. Müller, 1776 & A1, A2, A3, B1 (A, B) & $13,14,15,30,32,38,39,40,41,42$ \\
\hline L. lunaris Ehrenberg, 1832 & A3 (A) & $3,9,23,24,25,29$ \\
\hline L.nana Murray, 1913 & A5, B2 (A, B) & $13,14,15,39,30,32,38,39,40,41,42$ \\
\hline L.quadridentata Ehrenberg, 1832 & A5 (A) & $13,14,15,31,32,38,39,40,41,42$ \\
\hline L.obtusa Hauer, 1889 & A5 (A) & $2,13,14,15,31,32,38,39,40,41,42$ \\
\hline L. stenroosi Meissner, 1908 & A5 (A) & $13,14,15,31,32,38,39,40,41,42$ \\
\hline \multicolumn{3}{|l|}{ Lepadella Bory de St. Vincent, 1826} \\
\hline L. acuminate Ehrenberg, 1834 & A5 (A) & $13,14,15,31,32,38,39,40,41,42$ \\
\hline L.ehrenbergii Perty, 1850 & A5 (A) & $13,14,15,31,32,38,39,40,41,42$ \\
\hline L.ovalis O.F. Müller, 1786 & $\mathbf{A 2}, \mathbf{A 5}(\mathrm{A})$ & $13,14,15,31,32,38,39,40,41,42$ \\
\hline L.patella O.F. Müller, 1773 & $\mathbf{A 2}, \mathbf{A 3}, \mathbf{A 5}(\mathrm{A})$ & $13,14,15,39,30,32,38,39,40,41,42$ \\
\hline L.rhomboides Gosse, 1886 & A5 (A) & $1,2,13,14,15,31,32,38,39,40,41,42$ \\
\hline L.triptera Erenberg, 1830 & $\mathbf{A 2}, \mathbf{A 3}, \mathbf{A 5}(\mathrm{A})$ & $13,14,15,39,30,32,38,39,40,41,42$ \\
\hline \multicolumn{3}{|l|}{ Lophocharis Ehrenberg, 1838} \\
\hline L.oxysternon Gosse, 1851 & A3, A5 (A) & $13,14,15,39,30,32,38,39,40,41,42$ \\
\hline L. salpina Ehrenberg, 1834 & $\mathrm{A5}, \mathrm{A6}(\mathrm{A})$ & $13,14,15,31,32,38,39,40,41,42$ \\
\hline \multicolumn{3}{|l|}{ Monommata Bartsch, 1870} \\
\hline M. aequalis Ehrenberg, 1832 & A5 (A) & $13,14,15,31,32,38,39,40,41,42$ \\
\hline \multicolumn{3}{|l|}{ Mytilina Bory de St. Vincent, 1826} \\
\hline M. crassipes Luchs, 1912 & $\mathbf{A 5}(\mathrm{A})$ & $1,2,13,14,15,31,32,38,39,40,41,42$ \\
\hline M. mucronata Ehrenberg, 1832 & A5 (A) & $13,14,15,31,32,38,39,40,41,42$ \\
\hline $\begin{array}{l}\text { M. ventralis brevispina } \text { Ehrenberg, } \\
1832\end{array}$ & $\mathbf{A 2}, \mathbf{A 3}(\mathrm{A})$ & $13,14,15,39,30,32,38,39$ \\
\hline
\end{tabular}




\begin{tabular}{|c|c|c|}
\hline $\begin{array}{l}\text { M. ventralis ventralis Ehrenberg, } \\
1832\end{array}$ & $\mathbf{A 2}, \mathbf{A 3}(\mathrm{A})$ & $13,14,15,39,30,32,38,39$ \\
\hline \multicolumn{3}{|l|}{ Notholca Gosse, 1886} \\
\hline N. acuminatae Ehrenberg, 1832 & $\mathbf{A 2}, \mathbf{A 3}(\mathrm{A})$ & $13,14,15,39,30,32,38,39$ \\
\hline N. foliacea Ehrenberg, 1838 & A5 (A) & $1,2,13,14,15,31,32,38,39,40,41,42$ \\
\hline N. squamula O.F. Müller, 1786 & A2 (A) & $13,14,15,30,32,38$ \\
\hline \multicolumn{3}{|l|}{ Notommata Ehrenberg, 1830} \\
\hline N. copeus Ehrenberg, 1834 & $\mathbf{A 2}, \mathbf{A 3}, \mathbf{A 5}(\mathrm{A})$ & $13,14,15,39,30,32,38,39,40,41,42$ \\
\hline \multicolumn{3}{|l|}{ Philodina Ehrenberg, 1830} \\
\hline P. megalotrocha Ehrenberg, 1832 & A3 (A) & 36,37 \\
\hline \multicolumn{3}{|l|}{$\begin{array}{l}\text { Plationus Segers, Murugan\& } \\
\text { Dumont, } 1993\end{array}$} \\
\hline P.patulus O.F. Müller, 1786 & A2, A5 (A) & $13,14,15,39,30,32,38,39,41,42$ \\
\hline \multicolumn{3}{|l|}{ Platyias Harring, 1913} \\
\hline P. quadricornis Ehrenberg, 1832 & A3, A5 (A) & $13,14,15,39,30,32,38,39,41,42$ \\
\hline \multicolumn{3}{|l|}{ Ploesoma Herrick, 1885} \\
\hline P.hudsoni Imhof, 1891 & A5 (A) & $13,14,15,39,38,39,40,41,42$ \\
\hline P.truncatum Levander, 1894) & A3, A4, A5, B1 (A, B) & $13,14,15,39,30,32,38,39,41,42$ \\
\hline \multicolumn{3}{|l|}{ Polyarthra Ehrenberg,1834 } \\
\hline P.dolichoptera Delson, 1925 & A5 (A) & $13,14,15,31,32,38,39,41,42$ \\
\hline P. euryptera Wierzejski, 1891 & $\mathbf{A 5}(\mathrm{A})$ & $13,14,15,31,32,38,39,41,42$ \\
\hline P.major Bueckhardt, 1900 & A5, A5, B1, B2 (A, B) & $13,14,15,39,30,32,38,39,41,42$ \\
\hline P.minor Voigt, 1904 & $\mathrm{A5}(\mathrm{A})$ & $13,14,15,31,32,38,39,40,41,42$ \\
\hline P.remata Skorikov, 1896 & A5, B1, B2, E1 (A, B, E) & $2,13,14,15,39,30,32,38,39$ \\
\hline P.trygla Ehrenberg, 1834 & $\mathbf{A 1}, \mathbf{A 2}, \mathbf{A 3}, \mathbf{A 5}, \mathrm{B} 1(\mathrm{~A}, \mathrm{~B})$ & $13,14,15,39,30,32,38,39,41,42$ \\
\hline P.vulgaris Carlin, 1943 & $\mathbf{A 1}, \mathbf{A 2}, \mathbf{A 3}, \mathbf{A 5}, \mathrm{B} 1(\mathrm{~A}, \mathrm{~B})$ & $13,14,15,39,30,32,38,39,41,42$ \\
\hline \multicolumn{3}{|l|}{ Pompholyx Gosse, 1851} \\
\hline P.complanata Gosse, 1851 & A5, A6 (A) & $2,13,14,15,39,30,32,38,39$ \\
\hline P.sulcata Hudson, 1885 & B1, B2, D, E1, E2 (B, D, E) & $26,27,28,29,30,31,32$ \\
\hline P. triloba Pejler, 1957 & $\operatorname{A5}(\mathrm{A})$ & $13,14,15,31,32,38,39,41,42$ \\
\hline \multicolumn{3}{|l|}{ Rotaria Scopoli, 1777} \\
\hline R. citrine Ehrenberg, 1838 & A5 (A) & $13,14,15,31,32,38,39,41,42$ \\
\hline R. rotatoria Pallas, 1766 & A3, A5 (A) & $13,14,15,30,32,38,39,41,42$ \\
\hline R. socialis Kellicot, 1888 & A5 (A) & $13,14,15,31,32,38,39,41,42$ \\
\hline \multicolumn{3}{|l|}{ Scaridium Ehrenberg, 1830} \\
\hline S. longicaudum O.F. Müller, 1786 & $\mathbf{A 3}(\mathrm{A})$ & $7,8,35,36,37$ \\
\hline \multicolumn{3}{|l|}{ Squatinella Bory de St. Vincent, 1826} \\
\hline S. lamellaris O.F. Müller, 1786 & $\mathbf{A 5}(\mathrm{A})$ & $13,14,15,31,32,38,39,40,41,42$ \\
\hline \multicolumn{3}{|l|}{ Synchaeta Ehrenberg, 1832} \\
\hline S. littoralis Rousselet, 1902 & $\mathbf{A 5}, \mathbf{A 6}, \mathbf{E} 1(\mathrm{~A}, \mathrm{E})$ & $13,14,15,29,30,32,38,39,40,41,42$ \\
\hline S. pectinata Ehrenberg, 1832 & $\begin{array}{l}\mathrm{A} 1, \mathrm{A2}, \mathrm{A5}, \mathrm{A6}, \mathrm{B} 1, \mathrm{B2}, \mathrm{C1}, \mathrm{C} 5 .(\mathrm{A}, \\
\mathrm{B}, \mathrm{C})\end{array}$ & $\begin{array}{l}2,3,4,6,7,8,9,10,12,13,14,15,28, \\
19,20,21,22,23,24,25,27,28,29,30, \\
31,32,38,39,41,42\end{array}$ \\
\hline S. stylata Wierzejski, 1893 & $\mathrm{~B} 1, \mathrm{~B} 2, \mathrm{C} 1, \mathrm{C} 2, \mathrm{C} 3, \mathrm{C} 4, \mathrm{C} 5(\mathrm{~B}, \mathrm{C})$ & $27,28,29,30,31,32$ \\
\hline \multicolumn{3}{|l|}{$\begin{array}{l}\text { Testudinella Bory de St. Vincent, } \\
1826\end{array}$} \\
\hline T. mucronata Gosse, 1886 & A5 (A) & $13,14,15,31,32,38,39,41,42$ \\
\hline T. patina Hermann, 1783 & $\mathbf{A 2}, \mathbf{A 3}(\mathrm{A}, \mathrm{B})$ & $13,14,15,30,32,38,39,41,42$ \\
\hline T.truncata Gosse, 1886 & $\operatorname{A5}(\mathrm{A})$ & $13,14,15,31,32,38,39,41,42$ \\
\hline \multicolumn{3}{|l|}{ Trichocerca Lamarck, 1801} \\
\hline T.bicristata Gosse, 1887 & $\mathbf{A 3}(\mathrm{A})$ & $5,6,7,8,35,36$ \\
\hline T.capucina Wie\&Zach, 1893 & $\begin{array}{l}\text { A1, A2, A3, A4, A5, A6, B1, B2, D, } \\
\text { C3, C4, C5 (A, B, C) }\end{array}$ & $\begin{array}{l}2,3,4,6,7,8,9,10,12,13,14,15,28, \\
19,20,21,22,23,24,25,27,28,29,30, \\
31,32,38,39,41,42\end{array}$ \\
\hline T.cylindrica Imhof, 1891 & $\mathrm{A1}, \mathbf{A 2}, \mathbf{A 5}(\mathrm{A})$ & $\begin{array}{l}2,28,19,20,21,22,23,24,25,27,28 \\
29,30,31,32,38,39,40,41,42\end{array}$ \\
\hline
\end{tabular}




\begin{tabular}{|c|c|c|}
\hline T. elongate Gosse, 1886 & A5 (A) & $13,14,15,31,32,38,39,41,42$ \\
\hline T.iernis Gosse, 1887 & A5 (A) & $13,14,15,31,32,38,39,40,41,42$ \\
\hline T.longiseta Schrank, 1802 & $\mathbf{A 1}, \mathbf{A 2}, \mathbf{A 3}(\mathrm{A})$ & $13,14,15,30,32,38,39,40,41,42$ \\
\hline T.myersi Hauer , 1931 & A5 (A) & $13,14,15,31,32,38,39,41,42$ \\
\hline T.porcellus Gosse, 1886 & $\mathbf{A 5}(\mathrm{A})$ & $13,14,15,31,32,38,39,41,42$ \\
\hline T.pusilla Lauterborn, 1898 & $\mathbf{A 2}, \mathbf{E 3}, \mathbf{E 4}(\mathrm{A}, \mathrm{E})$ & $3,4,6,7,8,9,10,12,13,14$ \\
\hline T.rattus O.F. Müller, 1776 & $\mathbf{A 5}(\mathrm{A})$ & $13,14,15,31,32,38,39,41,42$ \\
\hline T.rousseleti Voigt, 1902) & A5 (A) & $13,14,15,31,32,38,39,41,42$ \\
\hline T.similis Wierzejski, 1893) & A1, A2, A5 (A) & $\begin{array}{l}2,28,19,20,21,22,23,24,25,27,28, \\
29,30,31,32,38,39,40,41,42\end{array}$ \\
\hline T.stylata Gosse, 1851 & A5 (A) & $13,14,15,31,32,38,39,41,42$ \\
\hline T. tenuior Gosse, 1886 & A2 (A) & $13,14,15,30,32,38$ \\
\hline T.weberi Jennings, 1903 & A2, A3 (A) & $\begin{array}{l}2,28,19,20,21,22,23,24,25,27,28, \\
29,30,31,32,38\end{array}$ \\
\hline \multicolumn{3}{|l|}{ Trichotria Bory de St. Vincent, 1827} \\
\hline T. curta Skorikov, 1914 & A5 (A) & $13,14,15,31,32,38,39,41,42$ \\
\hline T. pocillum O.F. Müller, 1776 & A3, A5 (A) & $2,13,14,15,39,30,32,38,39,41,42$ \\
\hline T. tetractis Ehrenberg, 1830 & A3, A5 (A) & $2,13,14,15,39,30,32,38,39,41,42$ \\
\hline
\end{tabular}

\section{Legends for letter symbols and nearest central point in brackets}

A1 - Lake Micro Prespa (N:40.691447; E:21.028600), A2 - Lake Macro Prespa (N:40.794431; E:20.946263), A3 - Lake Ohrid (N:40.974442; E:20.676057),

A4 - Lake Fierza (N:42.090651; E:20.395832),

A5 - Lake Scadar/Shkodra (N:42.150502; E:19.395438),

A6 - River Buna (N:42.005522; E:19.456769),

B1 - Reservoir Bovilla (N:41.444056; E:19.893058),

B2 - Lake Tirana (N:41.310917; E:19.816143),

C1 - Lake Belshi (N:40.978345; E:19.891313),

C2 - Lake Merhoe (N:40.952991; E:19.899225),

C3 - Lake Seferani (N:40.940781; E:19.920761),

C4 - Reservoir Thana (N:40.862995; E:19.840817),

C5 - Reservoir Murrizi (N:40.727543; E:19.728467),

D1 - Lake Butrinti (N:39.784623; E:20.032498),

E1 - Lagoon of Patok (N:41.631068; E:19.601154),

E2 - Lagoon of Karavasta (N:40.918227; E:19.475840),

E3 - Lagoon of Narta (N:40.538250; E:19.424175),

E4 - Lagoon of Orikum (N:40.317013; E:19.441460),

\section{Albanian water basin symbols in brackets from Dill} (1993)

A (Drini Basin); B (Ishmi/Erzeni Basin); C (Semani Basin); D (Coastal Ionian Sea); E (Coastal Adriatic Sea).

\section{List of numerical references}

1. Byron 1981, 2. Gannon et al. 1981, 3. Guseska et al. 2012, 4. Gusheska 2003, 5. Guseska et al. 2008, 6. Guseska et al. 2014, 7. Kostoski 1998, 8. Kostoski et al. 2004, 9. Kostoski et al. 2005, 10. Michaloudi et al. 1997, 11. Michaloudi 2005, 12. Milovanovic et al. 1965, 13. Nedeljkovic 1959, 14. Petković 1973, 15. Petković 1975, 16. Petković 1978, 17. Petković 1981, 18. Popovska-Stankovič 1988, 19. PopovskaStankovič 2003, 20. Serafimova-Hadžišče 1954, 21.
Serafimova-Hadžišče 1958, 22. Serafimova-Hadžišče 1978, 23. Shumka 1994, 24. Shumka 1998a, 25. Shumka 1998b, 26. Shumka et al. 1998, 27. Shumka 2000, 28. Shumka 2001, 29. Shumka et al. 2006, 30. Shumka 2014, 31. Shumka et al. 2018a, 32. Shumka et al. 2018b, 33. Tasevska 2005, 34. Tasevska et al. 2006, 35. Tasevska et al. 2008, 36. Tasevska et al. 2012a, 37. Tasevska et al. 2012b, 38. Tasevska et al. 2017, 39. Taseska et al. 2018, 40. Verščagin 1912, 41. Živkovic 1974, 42. Živkovic 1975.

Acknowledgements - The research carried in last ten years was funded by the Ministry of Science, Youth and Sports from the Republic of Albania (Grants No.: 11/2009) and NRC Norway through the project on Interdisciplinary Study of Large Balkan Lakes (2008-2012).

\section{REFERENCES}

BYRON, G.T. (1981): Growth rate of crustacean zooplankton in Skadar Lake. In. BEETON, A.M. \& KARAMAN, G.S. (Eds.) The biota and limnology of Lake Skadar, University Veljko Vlahović, Institute of Biological and Medicine Research Titograd, Montenegro, Yugoslavia, p. 217-219.

BRUSINA, S. (1898): Dreissensia torbari and other related palearctic species. Glasnik Hrvatskoga Naravoslovnoga Drustva, 10 (1/5): 197-208.

Cullaj, A., Hasko, A., Miho, A., Schanz, F. \& BRANDLE H. (2005): The quality of Albanian natural waters and the human impact. Environment International, 31: 133-146. doi: 10.1016/j.envint.2004.06.008

DE RIDDER, M. (1986): Annotated checklist of nonmarine rotifers (Rotifera) from African inland waters. Zoologische Documentatie, Koninklijk 
Museum voor Midden-Afrika, Tervuren, Belgium, 21: 5-123.

DE RIDDER, M. (1991): Additions to the "Annotated checklist of non-marine rotifers (Rotifera) from African inland waters". Revue d'Hydrobiologie tropicale, 24: 25-46.

DE RIDDER, M. (1993): Additions II to the "Annotated checklist of non-marine rotifers (Rotifera) from African inland waters". Biologisch Jaarboek Dodonaea, 61: 99-153.

DE RIDDER, M. \& SEGERS, H. (1997): Monogonont Rotifera recorded in the World literature (except Africa) from 1960 to1992. Studie documenten van het KBIN, 88, Brussels, Belgium.

DE SMET, W.H. (2006): Asciaporrectidae, a new family of Rotifera (Monogononta: Ploima) with description of Asciaporrecta arcellicola gen. et sp. nov. and $A$. difflugicola gen. et sp. nov. inhabiting shells of testate amoebae (Protozoa). Zootaxa, 1339: 31-49. doi: 10.11646/zootaxa.1339.1.2

DE SMeT, W.H. \& Pourriot, R. (1997): The Dicranophoridae (Monogononta) and the Ituridae (Monogononta). In. Nogrady T. (Ed.) Rotifera 5 In. DUMONT H.J. (Ed.) Guides to the Identificationof the Microinvertebrates of the Continental Waters of the World 12. SPB Academic, The Hague, The Netherlands, 344 pp.

DILL, W.A. (1993): Inland fisheries of Europe. EIFAC Technical Paper, No. 52 Suppl. Rome: FAO.

DONNER, J. (1965): Ordnung Bdelloidea (Rotatoria, Rädertiere). Bestimmungsbücher zur Bodenfauna Europas, 6: 1-267.

DUMONT, H. (1983): Biogeography of rotifers. Hydrobiologia, 104:19-30. doi: 10.1007/978-94-0097287-2 4

Ferrara, O., VAGAGgini, D. \& MARGARITORA, F.G. (2002): Zooplankton abundance and diversity in Lake Bracciano, Latium, Italy. Journal of Limnology, 61(2): 169-17. doi: $\underline{10.4081 / j \text { limnol.2002.169 }}$

Fontaneto, D., De Smet, W.H. \& Melone, G. (2008): Identification key to the genera of marine rotifers worldwide. Meiofauna Marina, 16: 75-99.

GANNON, J. \& STEMBERGER, R. (1981): Impact of influent rivers in the distribution of zooplankton in Skdar Lake. In. BEETON, A.M. \& KARAMAN, G.S. (Eds.) The biota and limnology of Lake Skadar,
University Veljko Vlahović, Institute of Biological and Medicine Research Titograd, Montenegro, Yugoslavia, p. 199-216.

GUSESKA, D. (2003): Comparative morphologicalecological characteristics of Calanoida and Cyclopoida (Crustacea; Copepoda) from Lake Ohrid pelagic zone. $\mathrm{PhD}$ dissertation, University "St. Cyril and Methodius", Skopje, R.Macedonia, 211 pp.

Guseska, D., Kostoski, G \& TASevskA, O. (2008): Seasonal successions of zooplankton in the pelagic zone of Lake Prespa during 2004-2006. In. Proceedings of BALWOIS 2008 - Ohrid, Republic of Macedonia.

Guseska, D., TAsevskA, O. \& Kostoski, G. (2012). Zooplankton dynamic of Lake Prespa (Macedonia). Biologia, 67(5): 939-944. doi:10.2478/s11756-012$\underline{0092-\mathrm{Z}}$

GuŠEska, D., Tasevska, O., Kostoski, G. \& GUSESKI, D. (2014): Zooplankton abundance and diversity in Lake Ohrid, Macedonia. International Journal of Ecosystems and Ecology Sciences, 4 (3): 333-340.

HOFFMANN, W. (1977): The influence of abiotic environmental factors on population dynamics in planktonic rotifers. Arch für Hydrobiologie, 8:7783.

JERSABEK, C.D. (2003): Freshwater Rotifera (Monogononta) from Hawai'i - a preliminary checklist. Bishop Museum occasional papers, 74: 46-72.

JeRsabeK, C.D. \& LEITNER, M.F. (2013): The Rotifer World Catalog. World Wide Web electronic publication. http://www.rotifera.hausdernatur.at/

KIEFER, F. (1937): Die freilebenden Ruderfusskrebse (Crustacea,Copepoda) Jugoslawiens. Bulletin de la Société Scientifique de Skoplje, 48: 77-105.

Kostoski, G. (1998): A Day-Night and seasonal periodicity in the distribution of the zooplankton from Lake Ohrid. PhD dissertation, University "St. Cyril and Methodius", Skopje, R. Macedonia, 180 pp.

Kostoski, G., GuŠESKA, D. \& TASEVSKA, O. (2004): A day-night and seasonal periodicity in the distribution of the zooplankton from Lake Ohrid. In. Proceedings of BALWOIS 2004 Conference of water observation and information system for decision support, Ohrid, R. Macedonia. 
Kostoski, G., GuŠEsKA, D. \& TASEVsKA, O. (2005): Zooplankton research in the Lake Ohrid pelagic region. Plankton investigations. Hydrobiological Institute, Ohrid, Limnological investigations of Ohrid and Prespa lakes, 3(4): 79-87.

Koste, W. (1978): Rotatoria. Die Rädertiere Mitteleuropas. Borntraeger, Berlin, Stuttgart, 2 vols, 673 pp.

Koste, W. \& SHIEL, R.J. (1987): Rotifera from Australian Inland waters II. Epiphanidae and Brachionidae (Rotifera: Monogononta). Invertebrate Taxonomy, 7: 949-1021. doi: 10.1071/IT9870949

Koste, W. \& ShIEL, R.J. (1989a): Rotifera from Australian Inland waters III. Euchlanidae, Mytilinidae and Trichotriidae. Transactions of the Royal Society of South Australia, 113(1-2): 85-114.

Koste, W. \& SHIEL, R.J. (1989b): Rotifera from Australian Inland waters IV. Colurellidae (Rotifera: Monogononta). Transactions of the Royal Society of South Australia, 113(3): 119-143.

Michaloudi, E., ZARFdJiAn, M. \& ECONOMIDIS, P.S. (1997): The Zooplankton of Lake Mikri Prespa. Hydrobiologia, 351: 77-94. doi: $\underline{10.1023 / \mathrm{A}: 1003008306292}$

MichalOUDI, E. (2005): Dry weights of the zooplankton of Lake Mikri Prespa (Macedonia, Greece). Belgian Journal of Zoology, 135: 223227.

Milovanovic, D. \& ŽIvkovic, A. (1965): Plankton Skadarskog jezera (1957 do 1958). Zbornik radova Biloškog Instituta, Beograd, 8: 23-29.

NEDELJKOVIC, R. (1959): Skadarsko jezero: studija organske prodikcije u jednom kaesnomjezeru. Posebno izdavanje Biolskog Instituta, Beograd, 4: 1-56.

Nogrady, T. \& SEgers, H. (2002): Rotifera 6. The Asplanchnidae, Gastropodidae, Lindiidae, Microcodinidae, Synchaetidae, Trochosphaeridae. In: DuMONT, H.J. (Ed.) Guides to the Identification of the Microinvertebrates of the Continental Waters of the World 18. Backhuys Publishers BV, Dordrecht, The Netherlands, 264 pp.

Nogrady, T., Pourriot, R. \& Segers, H. (1995): Rotifera 3. The Notommatidae and The Scaridiidae. In: DuMONT H.J. (Ed.) Guides to the Identification of the Microinvertebrates of the Continental Waters of the World 8.SPB Academic, The Hague, The Netherlands, 248 pp.
PARENZAN, P. (1931): CladoceriD‘Albaniacon brevi notizie morfologische ed hidrobiologische et idrobiologische sui grandilaghialbanesi. Atti della Academia Veneto Trentino, Istriana, 22: 16--47.

PetKović, S. (1973): Prilog poznavanju faune Rotatoria Crne Gore IIa.-Fam.Trichotriidae. Glasnik Repubike Zavoda Zaštite Prirode - Prirodnjackog muzeja Titograd, 2: 129-134.

PetKović, S. (1975). Prilog poznavanju faune Rotatoria Crne Gore IIb. Fam. Brachionidae, genera: Notholca, Kellicottia, Argonotholca i Anuraeopsis. Poljoprivreda $i$ šumarstvo, 21(4): 59-79.

PetKović, S. (1978): The contribution to the better knowledge of ecology and distribution of Brachionus sessilis Varga, 1957 (Rotatoria). Poljoprivreda $i$ šumarstvo, 24(2): 17-37.

PetKović, S. (1981): Zooplankton - General introduction. In: BEETON AM, KARAMAN GS. (Eds.) The biota and limnology of Lake Skadar, University Veljko Vlahović, Institute of Biological and Medicine Research Titograd, Montenegro, Yugoslavia, p. 191-192.

PetKovski, T. (1961): Zur Kenntnis der Crustaceen des Skadar (Scuteri ) Sees. Acta Musei Macedonici Scientiarum Naturalium, 8(2): 29-52.

POPOVSKA-STANKOVIČ, O. (1988): Arctodiaptomus steindachneri (Richard 1987), (Crustacea, Copepoda) in Lake Prespa. In: Proceedings of the Third Congress of Ecologists of Yugoslavia,Ohrid, Yugoslavia, p. 308 .

Popovska-Stankovič, O., Kostoski, G., Guseska, D. \& TASEVSKA, O. (2003): Temporal successions of most important zooplankton populations of Lake Prespa. In: Proceedings of the $32^{\text {th }}$ Annual Conference of the Yugoslav Water Pollution Control Society "Water 2003", Zlatibor, Yugoslavia, p. 201-206.

Preston, N.D. \& RUSAK, J.A. (2010): Homage to Hutchinson: does inter-annual climate variability affect zooplankton density and diversity? Hydrobiologia, 653:165-177. doi: 10.1007/s10750$\underline{010-0352-2}$

RICHARD, J. (1897): Entomostraces recueillis par M. le Directeur Steindachner dans les lacs de Janina et Scutari. Annalen des K. K. Naturhistorischen Hofmuseums in Wien, Serie B, Botanik und Zoologie, 12: 63-66. 
SEGERS, H. (1995a): Rotifera 2. The Lecanidae (Monogononta). In: DuMONT, H.J. (Ed.) Guides to the Identification of the Continental Waters of the World 6. SPB Academic, The Hague, The Netherlands, $226 \mathrm{pp}$.

SEGERS, H. (1995b): World records of Lecanidae (Rotifera: Monogononta). Studiedocumenten van het Koninklijk Belgisch Instituutvoor Natuurwetenschappen, 81: 1-114.

SEGERS, H. (2002): The nomenclature of the Rotifera: annotated checklist of valid family- and genusgroup names. Journal of Natural History, 36: 631-640. doi: $\underline{10.1080 / 0022293023173339707}$

SEGERS, H. (2003): A biogeographical analysis of rotifers of the genus Trichocerca Lamarck, 1801 (Trichocercidae, Monogononta, Rotifera), with notes on taxonomy. Hydrobiologia, 500: 113-114. doi: 10.1023/A:1024624132386

Segers, H. \& Wallace R.L. (2001): Phylogeny and classification of the Conochilidae (Rotifera: Monogononta). Zoologica Scripta, 30(1), 37-48. doi: 10.1046/j.1463-6409.2001.00048.x

Segers, H., DE SMet, .W.H., Fischer, C., FonTANETO, D \& MichalOUDI, E. (2012): Towards a list of available names in zoology, partim phylum Rotifera. Zootaxa, 3179: 61-68. doi: 10.11646/zootaxa.3179.1.3

SERAFIMOVA-HADŽIŠČE, J. (1954): Vertikalni migracii na zooplanktonot vo Prespanskoto Ezero. Zbornik na Rabotite Hidrobiološki Zavod, 1: 29-38.

SERAFIMOVA- HADŽIŠČE, J. (1958): Particularités du zooplankton du lac de Prespa et apercu de la composition du zooplancton des grands lacs de la peninsule des Balkans. Zbornik na Rabotite Hidrobiološki Zavod, 12: 1-8.

SERAFIMOVA- HADŽIŠČE, J. (1975): Seasonal variations of the reproductive rate of pelagic copepods of Lake Ohrid. Verhandlungendes Internatlen Verein Limnologie, 19: 2976-2982. doi: 10.1080/03680770.1974.11896402

Steuer, A. (1900): Die Diaptomiden des Balkans zugleich ein Beitrag zur Kenntnis des Dioptomus vulgaris Schmeil. Sitzungsberichte der Kaiserlichen Akademie der Wissenschaften. Mathematisch -Naturwissenschaftliche Classe Abt. 1, 109: 315335.
SHUMKA, S. (1994): Annual zooplankton dynamics of Lake Prespa. PhD, University "St. Cyril and Methodius", Skopje, Macedonai, $111 \mathrm{pp}$

SHUMKA, S. (1997a): Significance of Prespa zooplankton in lake ecosystem functioning. In: Proceedings of the Conference "Towards Integrated Conservation and Sustainable Development of Transboundary Macro and Micro Prespa Lakes", 1997, Korca, Albania.

SHUMKA, S. (1997b): Qualitative composition of zooplankton from Micro Prespa lake. In: Proceedings of the Conference "Towards Integrated Conservation and Sustainable Development of Transboundary Macro and Micro Prespa Lakes”, 1997, Korca, Albania.

Shumka, S., Thomollari, Z., Mali, S. \& Aleksi, P. (1998): Can Rotifers from the pelagic region be used as indicators of Lake trophy state? $27^{\text {th }}$ Annual Water Control pollution Conference 1998, Proceedings, Kotor, Montenegro.

SHUMKA, S. (2000): Zooplankton community as an indicator of Lake trophic state (Makro Prespa Lake). In: Proceedings of the International Symposium on Sustainable Deveopment of Prespa Region, 2000, Oteshevo, R. Macedonia.

SHUMKA, S. (2001): Feeding relations of Eudiaptomus gracilis (SARS) and influence on some parameters of their life cycle in Lake Ohrid. Verhandlungendes Internatlen Verein Limnologie, 27: 3708-3711. doi: $\underline{10.1080 / 03680770.1998 .11902523}$

ShumKA, S. \& Miнo, A. (2006): Data on Plankton community (Zooplankton and phytoplankton) in Drini course. In: Proceedings of BALWOIS 2006 Conference of water observation and information system for decision support, Ohrid, R. Macedonia.

SHUMKA, S. (2014): Rotifers in the Littoral Zone of Lake Shkodra/Skadar (Albania-Montenegro) as a tool for Determining Water Quality. International Research Journal of Biological Sciences, 3(3): 7177.

ShumKA, S. \& NiKLeKA, E. (2018): Diversity of Zooplankton and its Distribution in Karavasta Lagoon (central Albanian Adriatic). Proceedings of the $1^{\text {st }}$ International Conference of the Holistic approach to Environment. September $13^{\text {th }}-14^{\text {th }}$, 2018, Sisak, Republic of Croatia. 
SHUMKA, S., ŠPOLJAR, M. \& TASEVSKA, O. (2018): The Zooplankton of Lake Skadar/Shkodra: Species Diversity and Abundance. PEŠIĆ, V., KOSTIANOY, A. G., KARAMAN, G. S. (Eds.) The Skadar/Shkodra Lake Environment. Springer, Berlin, pp. 223-241.

Sørensen, M.V., SEgers, H. \& FunCH, P. (2005): On a new Seison Grube, 1859 from coastal waters in Kenya, with a reappraisal of the classification of Seisonida (Rotifera). Zoological Studies, 44: 34-43.

TASEVSKA, O. (2002): Eco-taxonomic investigations of rotifers from Lake Ohrid. Master thesis, University 'St. St. Cyril and Methodius'. Skopje, R. Macedonia, $240 \mathrm{pp}$.

TAsevskA, O., Kostoski, G. \& GUSEsKA, D. (2006): Recent species composition of Rotifera fauna of the Lake Dojran. BALWOIS 2006 - Conference of water observation and information system for decision support, Ohrid, R. Macedonia.

Tasevska, O., Kostoski, G.\& Guseska, D. (2008): Composition and dynamics of planktonic rotifers in Lake Ohrid, Macedonia. Hydrobiological Institute Ohrid, Review, 41(1): 109-115.

TAsevska, O., Guseska, D. \& Kostoski, G. (2012a): Comparison of Pelagic Rotifer Communities in Three Natural Macedonian Lakes. Acta zoologica bulgarica, Supplement. 4: 159-165.

Tasevsk, O., JersabeK, C.D., Kostoski, G. \& GUŠESKA, D. (2012b): Differences in rotifer communities in two freshwater bodies of different trophic degree (Lake Ohrid and Lake Dojran, Macedonia). Biologia (Bratislava), 67(3): 565-572. doi: $10.1515 /$ cjf-2017-0013
TAseVsKa, O., ŠPOlJAR, M., GUŠESKA, D., Kostoski, G. \& PATCHEVA, S. (2017): Zooplankton in ancient and oligotrophic Lake Ohrid (Europe) in association with environmental variables. Croatian Journal of Fisheries, 75: 95-103. doi: 10.1515/cjf$\underline{2017-0013}$

TASEVSKA, O., ŠPOlJar, M., ShUMKA, S., KuCZYŃSKA-KIPPEN, N. \& DRAŽINA, D. (2018): Comparison of zooplankton functional response traits in deep lakes across Europe. Proceedings of the $13^{\text {th }}$ Croatian Biological Congress. with International Participation, 2018, Poreč, Croatia.

VEREŠČAGIN, G.J. (1912): Cladocera Skutarijskogo ozera (Cernogorija). Raboty iz laboratorii zoologicheskogo kabineta imperatorskago Varshavskago Universiteta, 2: 22-33.

Wallace, R.L., SNell, T.W. \& RicCI, C. (2006): Rotifera vol. 1: Biology, Ecology and Systematics (2nd edition). In: SEGERS, H. \& DUMONT, H.J. (Eds.) Guides to the Identification of the Microinvertebrates of the Continental Waters of the World, Volume 23. Kenobi productions, Ghent, Belgium and Backhuys Academic Publishing bv, The Hague, The Netherlands, 299 pp.

ŽIVKOvIC, A. (1974): Nova vrsta Rotatoria Platyias bicornis sp.n. u Skadarskom jezeru. Archiv bioloski nauka, 26(3-4): 193-195.

ŽIVKOVIC, A. (1975): Zooplankton and microfauna of the vegetative area of Skadar lake. In: Limnological Investiagations of Skadar Lake, Progress Report, Smithsonian Institution, p. 89-106. 